ISSN-L 2077-0014

DOI: https://doi.org/10.33326/26176068.2021.3.1153

Artículo original/Original article/Artigo original

\title{
Determinantes de elección de especialidad en estudiantes de medicina de Tacna
}

\author{
Determinants of specialty choice by medical students from Tacna \\ Determinantes da escolha da especialidade em estudantes de medicina de Tacna
}

\begin{abstract}
Anthony Velarde-Quispe ${ }^{1 a}$
Yudith Calahuille-Aycachi ${ }^{1 a}$

Eduardo Bolivar-Vargas ${ }^{1 a}$

Lisset Aduviri-Albarracín ${ }^{1 a}$

Diana Huanco Apaza ${ }^{\text {1b }}$
\end{abstract}

(iD) https://orcid.org/0000-0002-3272-8341

(D) https://orcid.org/0000-0002-3951-8902

(iD https://orcid.org/0000-0001-9734-7796

(D) https://orcid.org/0000-0001-8821-8079

(D) https://orcid.org/0000-0001-9687-8384

\begin{abstract}
Resumen
Objetivo: Determinar los factores que influyen en la elección de la especialidad en estudiantes de medicina de Tacna, Perú. Material y métodos: Estudio analítico transversal, en 110 estudiantes de medicina que cursaban de cuarto a séptimo año de estudio matriculados en el año 2020, en la Universidad Nacional Jorge Basadre Grohmann y Universidad Privada de Tacna. Se aplicó encuesta virtual anónima, previo consentimiento informado. Se utilizó estadístico Chi cuadrado para las comparaciones, mediante programa STATA 14. Resultados: La edad promedio de los estudiantes fue 23,6 \pm 2,7 años, 54,6 \% de género femenino, 89,1 \% de nivel socioeconómico medio, 94,6 \% proviene de Tacna, $62,7 \%$ no tiene familiares médicos y $94,6 \%$ sí tiene interés de estudiar una especialidad médica. 59,1 \% eligió una especialidad clínica; 38,2 \%, quirúrgica; y sólo 2,7 \%, administración y gerencia. Los principales motivos de elección fueron: ver casos interesantes y diversos $(85,5 \%)$, tener retos intelectuales $(83,6 \%)$, tener contacto cercano con el paciente $(82,7 \%)$, generar cambios en la comunidad (81,8 \%), y tener buenos ingresos económicos (78,2 \%). Entre las especialidades clínicas, prefieren pediatría (8,3 \%), cardiología $(7,3 \%)$, endocrinología $(6,4 \%)$ y medicina intensiva $(6,4$ $\%)$; de las quirúrgicas, traumatología $(7,3 \%)$, cirugía general $(6,4 \%)$, anestesiología $(4,6 \%)$ y cirugía plástica (4,6 \%). Como virtudes señalaron tener mayor afinidad al amor y bondad (73,6 \%) y a la integridad $(71,8 \%)$, y mayor destreza a la capacidad de comunicación con personas no expertas $(65,5 \%)$, capacidad de aplicar conocimientos a la práctica $(64,6$ \%) y razonamiento crítico (64,6 \%). Conclusiones: Existe mayor prevalencia en elegir especialidades clínicas como pediatría en estudiantes de medicina de Tacna. El apoyo a la comunidad y los desafíos que involucra una especialidad son las razones más importantes para elegir una especialidad médica.
\end{abstract}

\section{Palabras clave: especialidad, estudiantes de medicina, médicos}

\begin{abstract}
Objective: To determine the factors that influence the choice of specialty in medical students of Tacna, Peru. Material and methods: Cross-sectional analytical study in 110 medical students in the fourth to seventh year of study enrolled in the year 2020, at the Jorge Basadre Grohmann National University and Universidad Privada de Tacna. Anonymous virtual survey was applied, with prior informed consent. Chi-square statistic was used for comparisons, using STATA 14 program. Results: The average age of students was $23.6 \pm 2.7$ years, $54.6 \%$ female, $89.1 \%$ middle socioeconomic, 94.6 \% comes from Tacna, $62.7 \%$ has no medical relatives and $94.6 \%$ has an interest in studying a medical specialty. $59.1 \%$ chose a clinical specialty; $38.2 \%$, surgical; and only $2.7 \%$, administration and management. The main reasons for choice were: see interesting and diverse cases (85.5\%), have intellectual challenges (83.6 \%), have close contact with the patient $(82.7 \%)$, generate changes in the community $(81.8 \%)$, and have good economic income (78.2 \%). Among the clinical specialties, they prefer pediatrics $(8.3 \%)$, cardiology $(7.3 \%)$, endocrinology $(6.4 \%)$ and intensive medicine $(6.4$ $\%)$; surgical, traumatology (7.3 \%), general surgery (6.4\%), anesthesiology (4.6\%) and plastic surgery (4.6 \%). As virtues they pointed to have greater affinity to love and kindness $(73.6 \%)$ and integrity $(71.8 \%)$, and greater dexterity to the ability to communicate with non-experts (65.5\%), ability to apply knowledge to practice (64.6\%) and critical reasoning (64.6 $\%)$. Conclusions: There is a higher prevalence of choosing clinical specialties such as pediatrics in medical students of Tacna. Community support and the challenges involved in a specialty are the most important reasons for choosing a medical specialty.
\end{abstract}

Keywords: doctors, medical students, speciality

\footnotetext{
${ }^{1}$ Universidad Nacional Jorge Basadre Grohmann. Escuela de Enfermería. Tacna, Perú. 


\begin{abstract}
Resumo
Objetivo: Determinar os fatores que influenciam a escolha da especialidade em estudantes de medicina de Tacna, Peru. Material e métodos: Estudo analítico transversal, em 110 estudantes de medicina que cursavam do quarto ao sétimo ano de estudos matriculados no ano 2020, na Universidade Nacional Jorge Basadre Grohmann e Universidad Privada de Tacna. Pesquisa virtual anônima foi aplicada, com consentimento informado. Utilizou-se estatístico Quiquadrado para as comparações, mediante programa STATA 14. Resultados: A idade média dos estudantes foi 23,6 $\pm 2,7$ anos, 54,6 \% de género feminino, 89,1 \% de nível socioeconómico médio, 94,6 \% provém de Tacna, 62,7 \% não tem familiares médicos e 94,6 \% tem interesse em estudar uma especialidade médica. 59,1 \% escolheu uma especialidade clínica; 38,2 \%, cirúrgica; e apenas 2,7 \%, administração e gerência. Os principais motivos de escolha foram: ver casos interessantes e diversos (85,5\%), ter desafios intelectuais $(83,6 \%)$, ter contacto próximo com o paciente $(82,7 \%)$, gerar mudanças na comunidade $(81,8 \%)$ e ter bons rendimentos económicos $(78,2 \%)$. Entre as especialidades clínicas, preferem a pediatria (8,3\%), a cardiologia (7,3\%), a endocrinologia (6,4 \%) e a medicina intensiva $(6,4 \%)$, a cirurgia cirúrgica, a traumatologia $(7,3 \%)$, a cirurgia geral $(6,4 \%)$, a anestesiologia (4,6 \%) e a cirurgia plástica (4,6\%). Como virtudes assinalaram ter maior afinidade ao amor e bondade (73,6 \%) e à integridade (71,8 \%), e maior destreza à capacidade de comunicação com pessoas não peritas (65,5 \%), capacidade de aplicar conhecimentos à prática $(64,6$ \%) e raciocínio crítico $(64,6 \%)$. Conclusão: Existe maior prevalência em escolher especialidades clínicas como pediatria em estudantes de medicina de Tacna. O apoio à comunidade e os desafios que envolvem uma especialidade são as razões mais importantes para escolher uma especialidade médica.
\end{abstract}

Palavras-chave: especialidade, estudantes de medicina, médicos

\section{Introducción}

La elección de una especialidad médica, es un proceso complejo y dinámico, influenciado por diversos factores. Algunos autores han postulado que ciertos factores influyen en esta elección, como son: características personales, estilos de la vida asociados a la especialidad y características de las facultades de medicina, en el cual las expectativas están relacionadas con su futuro trabajo, inclusive antes de entrar en la licenciatura de medicina. ${ }^{1-3}$

Según una publicación de la Asociación Americana de Colegios Médicos sobre estadísticas detalladas de médicos activos en los Estados Unidos en el 2017, las especialidades con el mayor número de médicos activos de manera general fueron aquellas enfocadas en la atención primaria, especialidades de medicina interna, medicina familiar/medicina general, y pediatría. Por tanto, la tendencia de las especialidades médicas varía obedeciendo a las necesidades de los distintos especialistas que se requiera a nivel global, como regional. ${ }^{4-6}$

En un estudio realizado en Perú en el año 2016, sesenta y tres escuelas de medicina de Latinoamérica encontraron que un estilo de vida controlable es el factor predisponente en la elección de una especialidad médica, especialmente en el ámbito quirúrgico, pues dichas especialidades brindan más tiempo libre para dedicarse a la vida familiar y para el descanso, señalan también otros factores como el prestigio, el tiempo de formación, el entrenamiento requerido y la remuneración económica. ${ }^{4,7,8}$ En una encuesta nacional realizada a 1163 residentes en el año 2016 encontró que el $6,4 \%$ había renunciado anteriormente a una plaza de especialidad, los postulantes a la residencia son médicos recién graduados que tienen una visión más completa de las diferentes áreas que la medicina les ofrece, los cuales realizaron rotaciones en la mayoría de las especialidades médicas en el pregrado, un año de internado médico durante el pregrado y un año de servicio social en un establecimiento rural del primer nivel de atención..$^{9,10}$ Por ello, es de esperar que los médicos peruanos tengan limitada información sobre muchas de las especialidades. ${ }^{1,11}$

En Tacna hay escasez de médicos especialistas en ramas poco comunes (geriatras, infectólogos, oncólogos, endocrinólogos), por lo que en ocasiones los pacientes son derivados a ciudades como Lima o Arequipa para su tratamiento, no existen estudios que enfaticen en los factores relacionados en la elección de una especialidad. La investigación es justificada debido a la gran demanda de especialidades por parte de la población en los servicios de salud. El objetivo de este estudio fue determinar los factores que influyen en la elección de una especialidad en estudiantes de medicina de Tacna. 


\section{Material y métodos}

Se realizó un estudio analítico, de corte transversal. La población estuvo conformada por 268 estudiantes de medicina de la Universidad Nacional Jorge Basadre Grohmann y Universidad Privada de Tacna, matriculados en el segundo semestre académico del año 2020. La muestra estuvo conformada por 110 estudiantes, se incluyó a aquellos que cursaron entre cuarto y séptimo año de estudio, que aceptaron participar en el estudio, previo consentimiento informado. Se excluyó a los estudiantes que suspendieron temporalmente sus estudios y con cuestionarios incompletos.

Se elaboró un cuestionario mediante la plataforma de Google Forms, basado en dos cuestionarios previamente validados por los autores Alarcón-Ruiz et al. y Kremer et al. ${ }^{1,12}$

En el instrumento se incluyó información sobre características personales, económicas, virtudes y destrezas, clasificándose individualmente con valores del 1 al 5 según sus virtudes: liderazgo, bondad, prudencia, integridad y persistencia; y según sus destrezas: capacidad de análisis, aprendizaje autónomo, capacidad de aplicar conocimientos en la práctica, razonamiento crítico y capacidad de comunicación con personas inexpertas, preferencia e interés por alguna especialidad, motivación para la selección de las especialidades y experiencias que direccionan a una especialidad. Además, se consideró las siguientes variables para el análisis como: ingresos económicos, cantidad de guardias, baja presión laboral, posibilidades de presenciar casos interesantes y diversos, contacto cercano con pacientes, la posibilidad de generar cambios en la comunidad, retos intelectuales, investigación, reputación y estatus, recomendación de los padres o de otros médicos especialistas, la dificultad para el ingreso y las experiencias personales. Estas variables fueron calificadas de dos maneras: poco importante o muy importante. Consta de 29 preguntas de respuestas múltiples y abiertas con tiempo de duración aproximado de 4 minutos para responder.

Se contactó con los encuestados mediante sus delegados de aula y otros miembros de su promoción, luego se los invitó a participar mediante una encuesta virtual anónima por medio de las redes sociales, solicitando su previo consentimiento informado.

Para el análisis estadístico la base de datos se exportó en el programa Microsoft Excel, posteriormente, se hizo una revisión de la calidad de información. Además, se realizó la caracterización de la población mediante análisis univariado con medidas descriptivas como valores absolutos y relativos de cada una de las variables categóricas, y para las variables cuantitativas se utilizó medidas de tendencia central y de dispersión como promedio, desviación estándar, mediana y rangos intercuartílicos, según corresponda. El análisis fue realizado con el paquete estadístico STATA versión 14.0.

Los aspectos éticos que se consideraron fueron la confidencialidad de los participantes, no se consideró los datos de identificación y se solicitó consentimiento informado.

\section{Resultados}

Un total de 113 estudiantes respondieron la encuesta, de los cuales se excluyó a 3 por no haber respondido correctamente las preguntas y no brindar el consentimiento de participación, teniendo al final $110(97,35 \%)$ encuestas para el análisis. Entre los estudiantes incluidos, la edad osciló entre 19 y 38 años, con una media de 23,6 $\pm 2,7$ años, 54,6 $\%$ fueron mujeres, $97,3 \%$ se encuentran solteros, $89,1 \%$ percibe tener nivel socioeconómico medio, 94,6 \% provienen de Tacna, 47,3\% pertenecen a la Universidad Nacional Jorge Basadre Grohmann y $52,7 \%$ a la Universidad Privada de Tacna, 46,4 $\%$ se encuentran estudiando sexto año, $62,7 \%$ no tienen familiares médicos, 53,6 \% eligió estudiar medicina entre los 12 y 16 años; y 94,6 \% tiene interés por realizar una especialidad médica al terminar la carrera. Al evaluar la asociación entre el tipo de especialidad a la que piensan postular y las características sociodemográficas y académicas, no se encontró asociación (Tabla 1). 


\section{Tabla 1}

Características sociodemográficas y académicas de los estudiantes de medicina

\begin{tabular}{|c|c|c|c|c|}
\hline \multirow{3}{*}{ Variables } & Total & \multicolumn{3}{|c|}{ Tipos de especialidad } \\
\hline & $N=110$ & Clínica & Quirúrgica & $\mathrm{p}$ \\
\hline & & $\mathrm{N}=65(59,1)$ & $\mathrm{N}=42(38,2)$ & \\
\hline Edad & $23,6 \pm 2,7$ & $23,8 \pm 2,9$ & $23,3 \pm 2,2$ & 0,32 \\
\hline \multicolumn{5}{|l|}{ Género } \\
\hline Femenino & $60(54,6)$ & $39(60)$ & $19(45,2)$ & \multirow{2}{*}{0,13} \\
\hline Masculino & $50(45,4)$ & $26(40)$ & $23(54,8)$ & \\
\hline \multicolumn{5}{|l|}{ Estado civil } \\
\hline Soltero & $107(97,3)$ & $63(96,9)$ & $41(97,6)$ & 0,83 \\
\hline Casado o conviviente & $3(2,7)$ & $2(3,1)$ & $1(1,4)$ & \\
\hline \multicolumn{5}{|l|}{ Nivel socioeconómico } \\
\hline Alto & $2(1,8)$ & $1(1,5)$ & $1(2,4)$ & \\
\hline Medio & $98(89,1)$ & $58(89,2)$ & $37(88,1)$ & 0,95 \\
\hline Bajo & $10(9,1)$ & $6(9,2)$ & $4(9,5)$ & \\
\hline \multicolumn{5}{|l|}{ Lugar de procedencia } \\
\hline Tacna & $104(94,6)$ & $61(93,6)$ & $40(95,2)$ & 0,41 \\
\hline Moquegua & $2(1,8)$ & $2(3,1)$ & $0(0)$ & \\
\hline Puno & $2(1,8)$ & $1(1,5)$ & $1(2,4)$ & \\
\hline Arequipa & $1(0,9)$ & $1(1,5)$ & $0(0)$ & \\
\hline San Martín & $1(0,9)$ & $0(0)$ & $1(2,4)$ & \\
\hline \multicolumn{5}{|l|}{ Universidad } \\
\hline UNJBG & $52(47,3)$ & $28(43,1)$ & $23(54,8)$ & 0,24 \\
\hline UPT & $58(52,7)$ & $37(56,9)$ & $19(45,2)$ & \\
\hline \multicolumn{5}{|l|}{ Año de estudio } \\
\hline 4to año & $21(19,1)$ & $12(18,5)$ & $6(14,3)$ & 0,63 \\
\hline 5to año & $31(28,2)$ & $16(24,6)$ & $15(35,7)$ & \\
\hline 6to año & $51(46,4)$ & $33(50,8)$ & $18(42,9)$ & \\
\hline 7mo año & $7(6,4)$ & $4(6,2)$ & $3(7,1)$ & \\
\hline \multicolumn{5}{|c|}{ Presencia de familiares médicos } \\
\hline Ninguna & $69(62,7)$ & $40(61,5)$ & $27(64,3)$ & 0,62 \\
\hline Tío/tía & $17(15,4)$ & $13(20)$ & $4(9,5)$ & \\
\hline Padre/madre & $8(7,3)$ & $4(6,2)$ & $4(9,5)$ & \\
\hline Primo/prima & $8(7,3)$ & $4(6,2)$ & $3(7,1)$ & \\
\hline Hermano/hermana & $8(7,3)$ & $4(6,2)$ & $4(9,5)$ & \\
\hline \multicolumn{5}{|c|}{ Edad en la que decidió estudiar medicina } \\
\hline Entre 6 y 11 años & $23(20,9)$ & $15(23,1)$ & $8(19,1)$ & 0,58 \\
\hline Entre 12 y 16 años & $59(53,6)$ & $32(49,2)$ & $25(59,5)$ & \\
\hline Entre 17 y 20 años & $28(25,5)$ & $18(27,7)$ & $9(21,4)$ & \\
\hline \multicolumn{5}{|c|}{ Interés en realizar especialidad al término de la carrera } \\
\hline Sí & $104(94,6)$ & $63(96,9)$ & $39(92,9)$ & 0,20 \\
\hline No & $2(1,8)$ & $0(0)$ & $2(4,8)$ & \\
\hline Aun no sé & $4(3,6)$ & $2(3,1)$ & $1(2,4)$ & \\
\hline
\end{tabular}


Según el tipo de especialidad médica a postular, $59,1 \%$ de los encuestados eligió una especialidad clínica, 38,2 \% eligió una especialidad quirúrgica, y sólo 2,7 \% eligió la especialidad de administración y gerencia. Entre las especialidades clínicas, las más preferidas fueron pediatría $(8,3$ $\%)$, cardiología $(7,3 \%)$, endocrinología $(6,4 \%)$ y medicina intensiva (6,4\%). Entre las especialidades quirúrgicas, las más preferidas fueron traumatología $(7,3 \%)$, cirugía general $(6,4 \%)$, anestesiología $(4,6$ $\%)$ y cirugía plástica $(4,6 \%)$ (Tabla 2$)$.

\section{Tabla 2}

Características sociodemográficas y académicas de los estudiantes de medicina

\begin{tabular}{|c|c|c|c|}
\hline \multicolumn{2}{|c|}{$\begin{array}{l}\text { Especialidad } \\
\text { clínica }\end{array}$} & \multicolumn{2}{|c|}{$\begin{array}{l}\text { Especialidad } \\
\text { quirúrgica }\end{array}$} \\
\hline Especialidad & n (\%) & Especialidad & n (\%) \\
\hline Pediatría & $9(8,3)$ & Traumatología & $8(7,3)$ \\
\hline Cardiología & $8(7,3)$ & Cirugía general & $7(6,4)$ \\
\hline Endocrinología & $7(6,4)$ & Anestesiología & $5(4,6)$ \\
\hline Medicina intensiva & $7(6,4)$ & Cirugía plástica & $5(4,6)$ \\
\hline Dermatología & $4(3,7)$ & $\begin{array}{l}\text { Cirugía de } \\
\text { de tórax y } \\
\text { cardiovascular }\end{array}$ & $4(3,7)$ \\
\hline Geriatría & $4(3,7)$ & Neurocirugía & $4(3,7)$ \\
\hline Gastroenterología & $3(2,8)$ & $\begin{array}{l}\text { Ginecología y } \\
\text { obstetricia }\end{array}$ & $2(1,8)$ \\
\hline Neonatología & $3(2,8)$ & Cirugía pediátrica & $2(1,8)$ \\
\hline Neurología & $3(2,8)$ & Oftalmología & $2(1,8)$ \\
\hline Reumatología & $3(2,8)$ & $\begin{array}{l}\text { Otorrinolaringo- } \\
\text { logía }\end{array}$ & $1(0,9)$ \\
\hline Oncología & $3(2,8)$ & Cirugía oncológica & $1(0,9)$ \\
\hline $\begin{array}{l}\text { Emergencia y } \\
\text { desastres }\end{array}$ & $2(1,8)$ & & \\
\hline Nefrología & $2(1,8)$ & & \\
\hline Medicina familiar & $1(0,9)$ & Otra especialidad & \\
\hline Medicina interna & $1(0,9)$ & $\begin{array}{l}\text { Adminsitración y } \\
\text { gerencia }\end{array}$ & $3(2,8)$ \\
\hline Neumología & $1(0,9)$ & & \\
\hline Pisquiatría & $1(0,9)$ & & \\
\hline Rehabilitación & $1(0,9)$ & & \\
\hline Infectología & $1(0,9)$ & & \\
\hline Medicina deportiva & $1(0,9)$ & & \\
\hline
\end{tabular}

*Porcentaje presentado según total de alumnos
Entre los motivos para elegir una especialidad médica, que fueron considerados como importantes o muy importantes en ambas especialidades con mayor frecuencia, fueron: cuando sean especialistas puedan ver casos interesantes y diversos $(85,5$ $\%)$, que una vez sea especialista tenga retos intelectuales $(83,6 \%)$, que puedan tener contacto cercano con el paciente $(82,7 \%)$, que puedan generar cambios en la comunidad $(81,8 \%)$, que les permita tener buenos ingresos económicos $(78,2$ $\%)$, que les permita hacer investigación en dicha área (70\%), que no tengan mucha presión laboral ni estrés $(60 \%)$ y que le permita tener reputación y mejor status $(55,4 \%)$. En la Figura 1 se muestra los motivos por especialidad médica, no existiendo diferencias significativas entre ambas $(p>0,05)$.

\section{Figura 1}

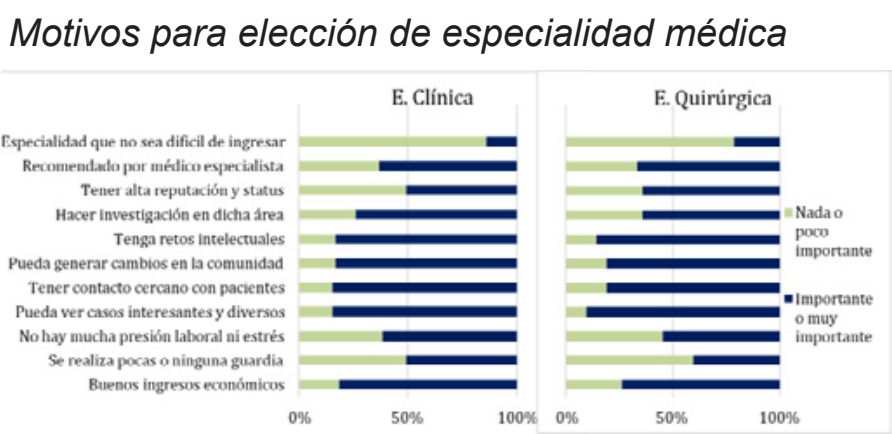

Los motivos de elegir una especialidad médica por universidad, resalta que las principales motivaciones los estudiantes de medicina de la Universidad Nacional Jorge Basadre Grohmann fueron tener retos intelectuales, poder ver casos interesantes y diversos; en cambio los estudiantes de la Universidad Privada de Tacna, se ven más motivados por generar cambios en la comunidad, además de ver casos interesantes. Se puede resaltar que los estudiantes de la universidad estatal tienen mayores motivaciones que la universidad privada por hacer investigación en dicha área y tener retos intelectuales; en cambio los estudiantes de medicina de la universidad privada tienen mayores motivaciones que los estudiantes estatales por generar cambios en la comunidad y que tengas buenos ingresos económicos (Figura 2). 


\section{Figura 2}

Motivos para elección de especialidad médica por universidad

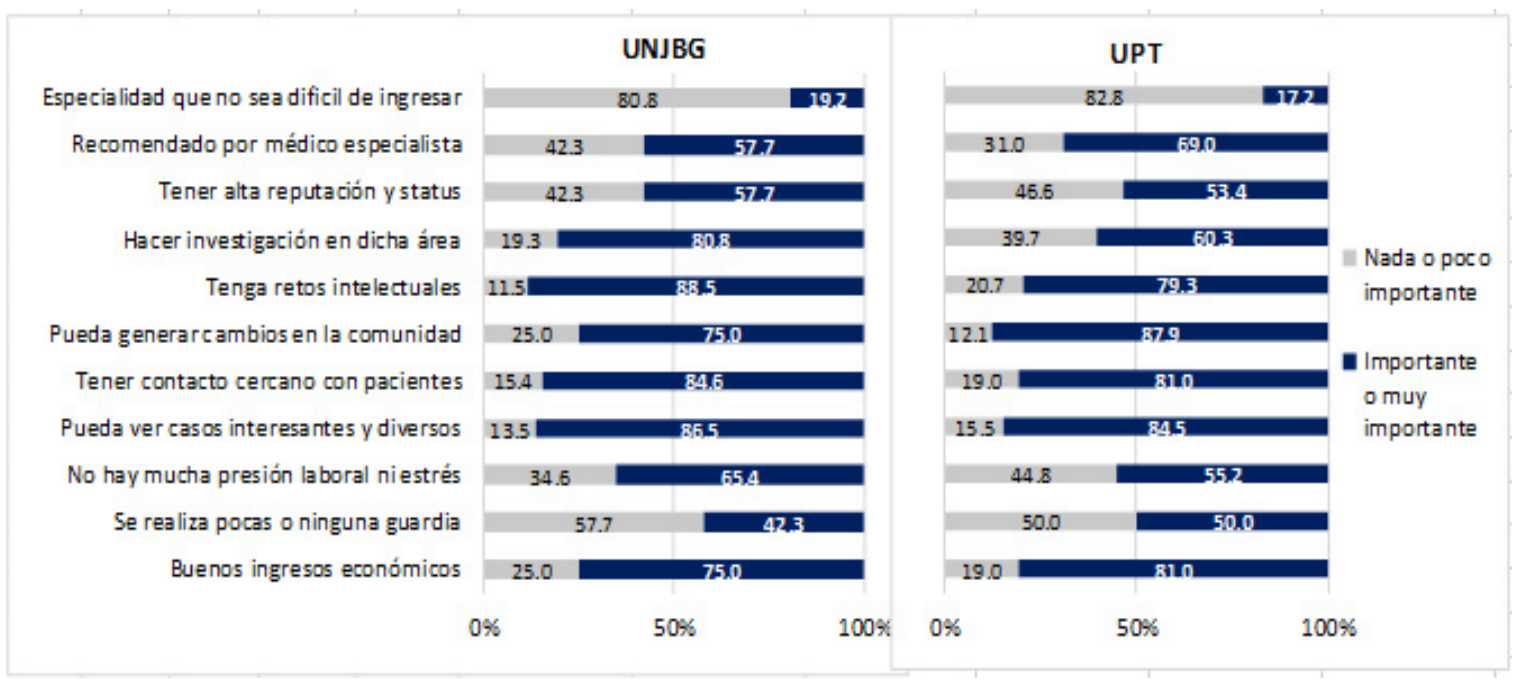

Los estudiantes de medicina calificaron individualmente cinco destrezas: capacidad de análisis y síntesis, aprendizaje autónomo, capacidad de aplicar conocimiento en la práctica, razonamiento crítico y capacidad de comunicación con personas no expertas, en base a una valoración de 1 a 5 , según

comunicación con personas no expertas $(65,5 \%)$ y capacidad de aplicar conocimiento en la práctica $(64,6 \%)$ y razonamiento crítico $(64,6 \%)$, en menor proporción a aprendizaje autónomo $(62,7 \%)$ y a la capacidad de análisis (60 \%) (Figura 3 ). la percepción propia de cada uno. Los resultados mostraron una mayor destreza a la capacidad de

\section{Figura 3}

Valoración de destrezas en estudiantes de medicina, según especialidad médica. De menor valoración (1) a mayor valoración (5)

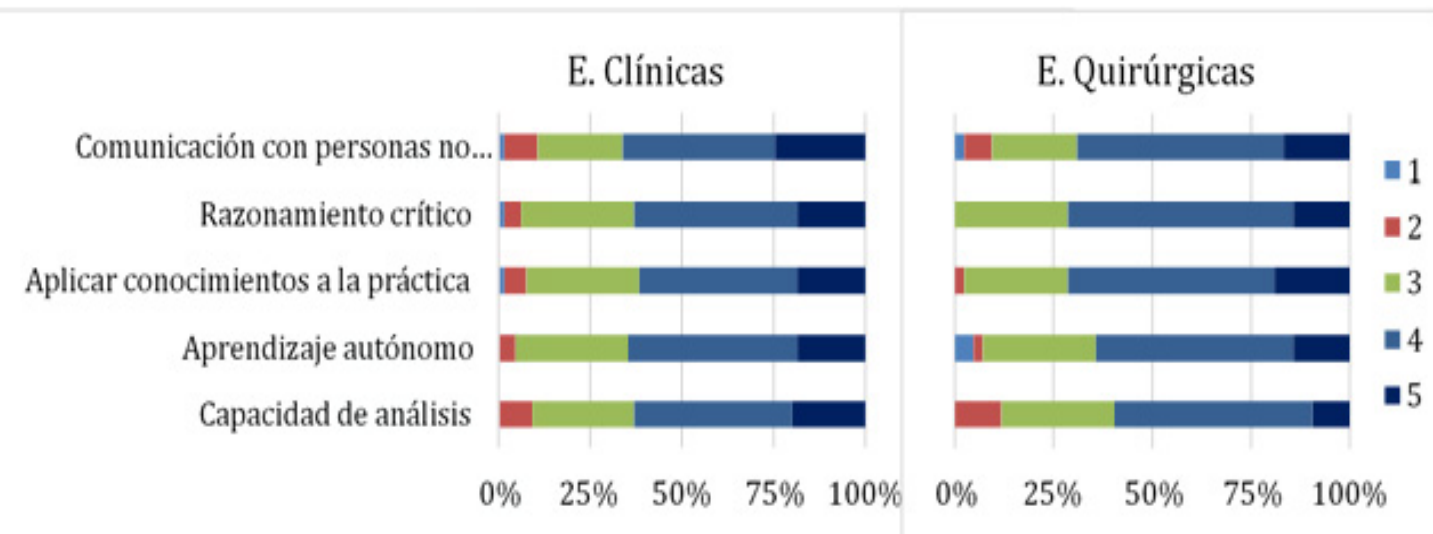

En los estudiantes de medicina se calificaron individualmente cinco virtudes: liderazgo, amor y bondad, prudencia, integridad, persistencia, en base a una valoración de 1 (menor) a 5 (mayor valoración), según la percepción propia de cada uno. Los resultados presentaron una mayor afinidad al amor y bondad $(73,6 \%)$ y a la integridad $(71,8 \%)$ y una menor afinidad al liderazgo (56,4 \%). Los estudiantes que elegirían especialidades clínicas tienen mayor afinidad al amor y bondad y aquellos que elegirían especialidades quirúrgicas tienen mayor afinidad a la integridad; sin embargo, no se encuentra asociación significativa entre la especialidad médica y las valoraciones de sus virtudes (Figura 4). 


\section{Figura 4}

Valoración de las virtudes en estudiantes de medicina según especialidad médica. De menor valoración (1) a mayor valoración (5)

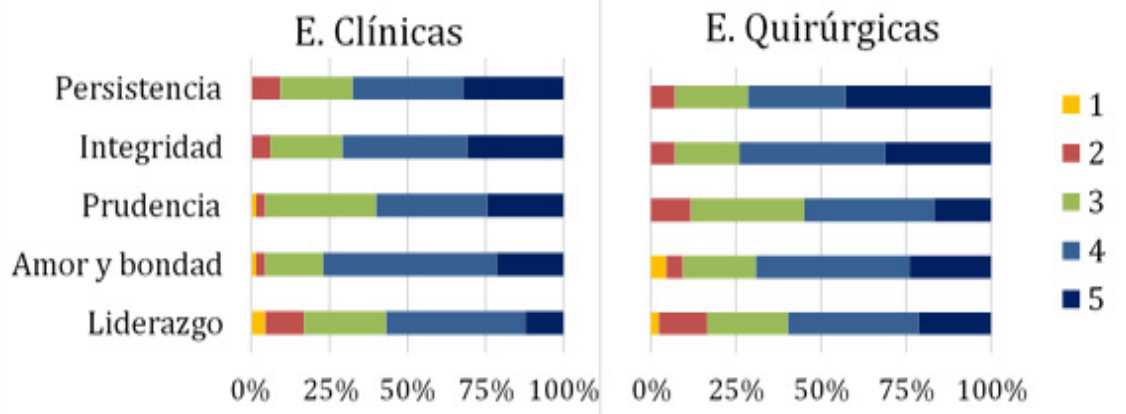

\section{Discusión}

De los estudiantes de medicina de Tacna participantes en este estudio, $57,8 \%$ y $37,6 \%$ se interesaron en elegir una especialidad clínica y quirúrgica, respectivamente. En las especialidades clínicas, las más frecuentes fueron pediatría, cardiología y medicina intensiva. Mientras, en especialidades quirúrgicas, fueron traumatología, cirugía general, anestesiología y cirugía plástica. Los factores que más influyeron en la elección de especialidad médica fueron generar cambios en la comunidad y afrontar desafíos intelectuales siendo especialistas. Además, se encontró a 6 estudiantes que relacionaron erróneamente el tipo de especialidad con la especialidad a escoger, esto demuestra que desconocen en qué rubro se encuentran, e implicaría de cierto modo una falta de información en los estudiantes.

En el estudio se evaluó las virtudes de los estudiantes, que es necesario para rescatar el humanismo del médico y una atención médica centrada en el bien del paciente. ${ }^{12}$ La mayor parte de estudiantes se caracterizaron por ser persistentes de los cuales mostraron preferencia por la especialidad de pediatría. Según Gutierrez-Cirlos C, los especialistas en medicina interna, ginecoobstetricia, y los estudiantes que eligen esta especialidad son más persistentes, conscientes, organizados. En cambio, los pediatras tienden a ser más del tipo ESTJ: extrovertidos, sensoriales, con pensamiento racional y con juicio calificador, de acuerdo con el indicador de Myers-Briggs. La dimensión corresponde más al neuroticismo (inestabilidad emocional), la extraversión y la amabilidad. ${ }^{13}$ Por tanto, uno de los factores que influyen en la elección de una especialidad es la personalidad y va correlativamente al desarrollo de virtudes.
Al evaluar también destrezas de los estudiantes, se tomó en cuenta la naturaleza de la carrera médica, la capacidad de análisis y síntesis que es mayormente requerida en algunas especialidades más que otras, lo que sería un factor importante en la elección de la especialidad las habilidades dominantes en cada estudiante. En el que la mayoría de los estudiantes que optan por una especialidad médica básica piensa que su actividad principal estará relacionada con esa especialidad, algunos consideraron otras actividades como otros posgrados, una carrera docente o permanecer como médicos generales. ${ }^{13}$

La prevalencia al elegir las carreras de pediatría y traumatología en el presente estudio fueron las mayores. Asimismo, la literatura ha reportado prevalencias altas de elección de estas especialidades, junto con oftalmología, gineco-obstetricia y anestesiología. ${ }^{1}$ De todos los estudiantes encuestados, el 94,55 \% mencionaron que sí tienen interés en realizar una especialidad clínica o quirúrgica al término de la carrera. Este resultado coincide con un estudio realizado en la Universidad Nacional San Luis Gonzaga, Ica-Perú, donde el $94 \%$ querían realizar una especialidad médica, mientras que solo el $3 \%$ deseaban ejercer como médico general. ${ }^{14}$ En 1951, Todd menciona que la proporción de especialistas aumentarán con los años, debido a que los pacientes deben ser atendidos por médicos a fines con la enfermedad del paciente. ${ }^{15}$ Por ello, se considera que la tendencia se mantendrá o incrementará en función a la elección de ser un médico especialista, siendo el médico general solo una etapa entre la graduación y especialización.

En el presente estudio, el $80 \%$ de los estudiantes consideró el factor económico como importante o muy importante, siendo el segundo 
de los motivos más influyentes para la elección de una especialidad. Estudios previos en residentes del Perú, mencionan que los factores económicos son considerados como los más importantes para la elección de una especialidad médica. ${ }^{1}$ Esto podría explicar, al menos parcialmente, el bajo índice de postulantes/vacantes en especialidades que son usualmente percibidas como de bajos ingresos económicos, como medicina familiar y comunitaria y medicina interna. ${ }^{15}$ Por otro lado, el factor considerado por el 87,5 \% como aún más importante que el económico fue el tener retos intelectuales o ver casos interesantes y diversos dentro de la especialidad. Esto es similar a los estudios realizados en estudiantes de medicina de Australia y Líbano donde se evidenció que los contenidos intelectuales tenían gran influencia en la elección de la especialidad..$^{1,17}$

En la región de Tacna hay 16,2 médicos por cada 10000 habitantes, siendo menor a lo recomendado por la Organización Mundial de la Salud (OMS) de 23 médicos por cada 10000 habitantes. En cuanto a la cantidad de especialistas en Tacna, no se tiene registro en zonas pobres, muy pobres y de extrema pobreza. ${ }^{18}$ Esto muestra la brecha de especialistas y la deficiente atención a las enfermedades específicas en la ciudad de Tacna, pudiendo ser los factores de esta realidad los deficientes recursos humanos, por la marcada asimetría en la distribución y proporción de los profesionales de la salud, y carencia de centros de atención primaria en los sectores pobres de la región. Sin embargo, se tiene la convicción de que los futuros profesionales lograrán cubrir las brechas de hoy en día.

Por otra parte, es importante mencionar que recibir recomendaciones de los padres, médicos o algún especialista, según este estudio, es poco o nada importante para los estudiantes. Estos resultados coinciden con estudios previos reportados en médicos residentes en Australia. ${ }^{17}$ Por lo tanto, las actividades informativas de especialidades podrían no influir en la elección final de los estudiantes, al menos que se mencione sobre los ingresos económicos, retos intelectuales, investigación de la especialidad y el grado de estrés laboral.

El estudio presenta algunas limitaciones. Al ser un estudio de tipo transversal, su evaluación se centró en la búsqueda de factores relacionados y no en el efecto causal de los datos recolectados. En cuanto a la recolección de datos, parte de la muestra de estudio presentó falta de disposición de tiempo para responder el cuestionario, como es el caso de los estudiantes de séptimo año de la carrera y falta de interés por responder el cuestionario proporcionado, por lo que no se contó con la cantidad de datos esperados. El ingreso económico fue analizado de manera categórica y no numérica, por lo cual no se puede evaluar adecuadamente la distribución del mismo. Otra dificultad fue hallar datos incompletos y erróneos en la encuesta, los cuales fueron descartados con objetividad del análisis final.

En conclusión, se encontró que el 94,55 \% de los estudiantes encuestados, tienen interés en realizar una especialidad al término de la carrera. Por otro lado, en Perú se está buscando potenciar la atención primaria, para lo cual se requiere de más médicos que laboren en este rubro. En el estudio se encuentra que hay una mayor prevalencia en elegir la especialidad de pediatría en los estudiantes de medicina de Tacna. El apoyo a la comunidad y los desafíos que involucra una especialidad a fin son los más importantes para la elección de una especialidad médica. Por lo tanto, se recomienda a los estudiantes de medicina que opten por elegir una especialidad de atención primaria con el fin de cubrir las carencias de especialistas en las zonas que necesitan de atención médica en la región de Tacna.

\section{Referencias}

1. Alarcón-Ruiz CA, Heredia P, Zafra-Tanaka JH, Taype-Rondan A. Motivos para la elección y preferencias de la especialidad médica en médicos generales del Perú. Acta Med Peru. 2020; 37(3):294-303. Doi: https://doi. org/10.35663/amp.2020.372.1063

2. Madrid A, Stephany A. Factores que influyen en la elección de la especialidad en internos de medicina del Hospital de Apoyo II-2 Sullana, 2018. Universidad Privada Antenor Orrego [Internet]. 2019 [citado 8 de enero de 2021]; Disponible en: https://repositorio.upao.edu.pe/ handle/20.500.12759/4753

3. Gutiérrez-Cirlos C, Naveja JJ, Sánchez-Mendiola M. Factores relacionados con la elección de una especialidad en medicina. Revista Investigación en Educación Médica [Internet]. 2017 [citado 8 de enero de 2021];6(23):206-14. Disponible en: http://riem.facmed.unam.mx:90/ojs/index.php/ riem/article/view/228

4. Vázquez AC, Martínez DC. Perspectiva de las estudiantes de medicina y especialistas frente a una rama quirúrgica asociada al género en Cuenca. 2020 [citado 8 de enero de 2021]; 
Disponible en: http://dspace.uazuay.edu.ec/ handle/datos/10238

5. Bitran CM, Zúñiga PD, Lafuente GM, Viviani GP, Mena CB. Influencia de la personalidad y el estilo de aprendizaje en la elección de especialidad médica. Revista médica de Chile [Internet]. octubre de 2005 [citado 8 de enero de 2021];133(10):1191-9. Disponible en: https://scielo.conicyt.cl/scielo.php?script=sci abstract\&pid=S0034-98872005001000008\&Ing =es \&nrm=iso\&tlng=es

6. AAMC [Internet]. Washington: AAMC; 2017 [actualizado 2020; citado 23 nov 2020]. Disponible en: https://www.aamc.org/datareports/workforce/interactive-data/activephysicians-sex-and-specialty-2017

7. AAMC [Internet]. Washington: AAMC; 2018 [actualizado 2020; citado 23 nov 2020]. Disponible en: https://www.aamc.org/system/ files/2019-08/2018executivesummary.pdf

8. Vereau S, Enrrique J. Factores asociados a la elección de una especialidad quirúrgica en estudiantes de sesenta y tres escuelas de medicina de Latinoamérica. Univ Priv Antenor Orrego [Internet]. 2016 [citado 4 de marzo de 2021]; Disponible en: https://repositorio.upao. edu.pe/handle/20.500.12759/2160

9. Escobedo-Palza S, Nieto-Gutierrez W, TaypeRondan A. Características del residentado médico en el Perú: resultados de la primera Encuesta Nacional de Médicos Residentes. Consejo Nacional de Residentado Médico. (ENMERE-2016). Acta Med Peru [Internet]. 2017 [Citado 24 noviembre del 2020]; 34(4):273-282. Disponible en: http:/l www.scielo.org.pe/scielo.php?script=sci arttext\&pid=S1728-59172017000400004

10. Padilla-Cuadra JI, Vindas-Sánchez L, VillalobosPérezA. Decisión de estudiar medicina: Factores determinantes y elección de la especialidad. Rev AMC. [Internet]. 2012 [4 de enero 2021]; 54 (2): 109-113. Disponible en: https://www.redalyc.org/ articulo.oa?id=43422619007

11. Puertas EB, Orellana RA, Muñoz BE, Jiménez JA, Medina Quiroz IG, Terrón L, et al. Factores que influyen en la elección de una carrera en atención primaria entre los estudiantes de medicina en América Central. Rev Panam Salud Publica. 2020; 44:1-10. Doi: https://doi. org/10.26633/RPSP.2020.94
12. BAIN LE. Revisiting the need for virtue in medical practice: a reflection upon the teaching of Edmund Pellegrino. Philos Ethics Humanit Med. 2018; 13 (1):4. Published 2018 Apr 10. doi:10.1186/s13010-018-0057-0

13. Gutiérrez-Cirlos C, Naveja-Romero J, Leenen I, Sánchez-Mendiola M. Factores relacionados con la elección de una especialidad en médicos residentes mexicanos. Gac Med Mex. 2017; 153(7):800-809. Doi: dx.doi.org/10.24875/ GMM.17002959

14. Avgerinos ED, Msaouel $P$, Koussidis GA, Keramaris NC, Bessas Z, Gourgoulianis K. Greek medical students' career choices indicate strong tendency towards specialization and training abroad. Rev Elsevier. 2006; 79:101-6. Doi: https://doi.org/10.1016/j.healthpol.2005.12.007

15. Todd JW. Specialization. Lancet 1951; 1:462-7. Doi: https://doi.org/10.1016/S01406736(51)92054-5

16. Taype-Rondan A, Torres-Roman JS, HerreraAnazco P, Diaz CA, Branez-Condorena A, Moscoso-Porras MG. Ingresos económicos en médicos peruanos según especialidad: Un análisis transversal de la ENSUSALUD 2015. Rev Peru Med Exp Salud Publica. 2017; 34(2):18391. Doi: 10.17843/rpmesp.2017.342.2517.

17. Khater-Menassa B, Major S. Factors influencing the choice of specialty among medical students in Lebanon. J Med Liban. 2005 [13 de enero del 2021];53(1):16-20. Disponible en: https:// pubmed.ncbi.nlm.nih.gov/16398208/

18. COMEX - Sociedad de Comercio Exterior del Perú. RUMBO AL BICENTENARIO: RECURSOS HUMANOS DE SALUD [Internet]. [citado 28 de febrero de 2021]. Disponible en: https://www.comexperu.org.pe/articulo/rumboal-bicentenario-recursos-humanos-de-salud

19. Khan SM. Trends of Surgical Career Selection Among Medical Students and Graduates : A Global. JSURG [Internet]. 2010;67(4):237-48. Available from: http://dx.doi.org/10.1016/j. jsurg.2010.05.006

\section{Correspondencia:}

mvelardec@unjbg.edu.pe
Fecha de recepción: 25/06/2021

Fecha de aceptación: 09/08/2021 